\title{
EFECTO DE LA LIOFILIZACIÓN SOBRE LAS PROPIEDADES FUNCIONALES DEL AJí ROCOTO (Capsicum pubescens)
}

\section{EFFECT OF THE FREEZE - DRYING ON THE FUNCTIONAL PROPERTIES ROCOTO CHILI PEPPER (Capsicum pubescens)}

\author{
Birina Luz Caballero Gutiérrez ${ }^{1}$, Carlos Julio Márquez Cardozo², Benjamín Alberto Rojano ${ }^{3}$
}

\begin{abstract}
${ }^{1}$ Ingeniera Agroindustrial, Estudiante de Maestría en Ingeniería Agroindustrial. Universidad Nacional de Colombia, Calle 59A No.63-20, Medellín, Colombia, e-mail: blcaballerog@unal.edu.co; ${ }^{2}$ Ingeniero Agrícola, Ph.D. en Ciencias Agrarias, Profesor Asociado. Universidad Nacional de Colombia, Calle 59A No 63-20, Medellín, Colombia, e-mail: cjmarque@unal.edu.co; ${ }^{3}$ Químico, Ph.D. en Ciencias Químicas, Profesor Asociado. Universidad Nacional de Colombia, Calle 59A No 63-20, Medellín, Colombia, e-mail: brojano@unal.edu.co
\end{abstract}

Rev. U.D.C.A. Act. \& Div. Cient. 20(1): 111-119, Enero Junio, 2017

\section{RESUMEN}

El ají rocoto (Capsicum pubescens) es un fruto perecedero, por lo cual, se hace necesario explorar alternativas agroindustriales, que permitan conservar sus propiedades funcionales. Entre los desarrollos tecnológicos estudiados en la transformación y la conservación de alimentos, se encuentra la liofilización. En esta investigación, se liofilizó ají rocoto, colectado en San Cristóbal-Medellín, Antioquia (Colombia) y se evaluó su efecto sobre las propiedades funcionales, como la capacidad antioxidante, por el método Ferric Reducing Antioxidant Power (FRAP), fenoles totales, carotenoides, contenido de ácido ascórbico y capsaicina, tanto en el producto fresco como en los tratamientos liofilizados. Se realizó un arreglo de parcelas dividas, con el factor de la programación de la velocidad de calentamiento, entre 0,02 y $0,05^{\circ} \mathrm{C} /$ min, durante la sublimación, asignado a la parcela principal y al factor categórico de semilla en la subparcela, con una aleatorización completamente al azar (DCA), con tres repeticiones. Se registró, que el ají rocoto fresco con semilla incluida, la placenta valores de $1,18 \mathrm{mg}$, equivalentes de ácido ascórbico/g base seca (b.s.), como capacidad antioxidante, por FRAP; 5,37mg, equivalentes de ácido gálico/g b.s., para el contenido de fenoles totales; $4,74 \mathrm{mg} / \mathrm{g}$, b.s. de carotenoides; $1,88 \mathrm{mg} / \mathrm{g}$ b.s., de ácido ascórbico y $1,57 \mathrm{mg} / \mathrm{g}$ b.s., de capsaicina. Se concluyó que para los tratamientos liofilizados con y sin semillas, se incrementó la capacidad antioxidante y fenoles totales con respecto al producto en fresco. Se evidenció diferencia estadística en las programaciones de calentamiento durante la sublimación, afectando el contenido de capsaicina, en los tratamientos con y sin semillas.

Palabras clave: Antioxidantes, deshidratación, capsaicina, carotenoides, fenoles.

\section{SUMMARY}

The chili pepper (Capsicum pubescens) is a perishable fruit whereby it is necessary to explore agroindustrial alternatives, that preserves its functional properties; within the technological developments studied in food processing and preservation is freeze-drying. In this research, the rocoto chili pepper harvested in rural area of San CristobalMedellin, Antioquia (Colombia), was freeze-drying and it was evaluated the effect on the functional properties such as antioxidant capacity by the Ferric Reducing Antioxidant Power method (FRAP), total phenols, carotenoids, ascorbic acid and capsaicin, both in the fresh product and in freezedrying treatments. Was realized a fix split plots, with the factor of programming the heating rate, between 0.02 and $0.05^{\circ} \mathrm{C} / \mathrm{min}$ during sublimation assigned to the principal plot and the categorical factor seeds in the subplot, was performed with a Randomized Block Design (RBD) with three replications. It was obtained in fresh chilli with seed including placenta values of $1.18 \mathrm{mg}$ of eq. ascorbic acid $/ \mathrm{g}$ dried basis (d.b.) as antioxidant capacity by FRAP; $5.37 \mathrm{mg}$ of eq. gallic acid $/ \mathrm{g}$ d.b. for total phenols content; $4.74 \mathrm{mg} / \mathrm{g}$ d.b. of carotenoids; $1.88 \mathrm{mg} / \mathrm{g}$ d.b. of ascorbic acid and $1.57 \mathrm{mg} / \mathrm{g}$ d.b. of capsaicin. It was concluded that for the freeze-drying treatments, the antioxidant capacity and total phenols were increased, with respect to the fresh product. It evidenced a statistical difference in the heating schedules during sublimation affecting the capsaicin content in seed and seedless treatments.

Key words: Antioxidant, drying, capsaicin, carotenoids, phenols. 


\section{INTRODUCCIÓN}

El ají es una solanácea del género Capsicum, con centro de diversidad genética en Centro y Suramérica, extendido luego hacia toda América y Europa. Según Halikowski (2015), la pungencia es uno de los atributos más importantes en la calidad organoléptica del ají, lo cual, es atribuido al contenido de capsaicinoides, que varían entre 0,1 y $1 \%$, de acuerdo a la especie; la capsaicina (trans 8 metil-N-vanili-6-nonenamida), se encuentra entre el 50 y $70 \%$ e dihidrocapsaicina (8 metil-N-vanililnonanamida), del 20 y $25 \%$ de los capsaicinoides totales (Topuz et al. 2011). Algunas investigaciones han reportado que la capsaicina es una sustancia efectiva como tratamiento antitumoral, con propiedades antioxidantes (Bort et al. 2014). El grado de pungencia generado por los capsaicinoides, se expresa por la escala Scoville Heat Units (SHU), como prueba organoléptica, pero, actualmente, se utilizan métodos cuantitativos, como los de cromatografía líquida de alta resolución (HPLC), donde $1 \mathrm{mg} / 100 \mathrm{~g}$ de producto fresco equivale a 161 unidades SHU, para capsaicina o dihidrocapsaicina y, a 93 unidades SHU, para nordihydrocapsaicin (Meckelmann et al. 2015).

En términos de contribución nutricional, el ají picante contiene ácido ascórbico, que puede ser mayor a otros frutos (Cruz et al. 2007), al igual que de carotenoides, moléculas precursoras de la vitamina A (Rodríguez et al. 2010)capsanthin was the main carotenoid in the 3 species ( $25 \%$ to $50 \%$ contribution to carotenoid fraction. Su consumo promueve diversos beneficios, estimula la liberación de endorfinas, que permite aliviar los dolores reumáticos, mejora la circulación y ayuda a la digestión al estimular la saliva y el jugo gástrico (Sharma et al. 2013)transient receptor potential vanilloid subfamily member 1 (TRPV1 y posee componentes antioxidantes, de acuerdo a lo encontrado por Loizzo et al. (2015). Identificándose como un fruto con propiedades funcionales, puesto que posee componentes fisiológicamente activos, que propician beneficios en la salud (Reis et al. 2013; Simonovska et al. 2014).

La liofilización es una tecnología recomendada en la deshidratación de alimentos, que contienen componentes funcionales sensibles al calor, tales como tocoferoles, ácido ascórbico, carotenoides y fenoles (Shofian et al. 2011). El proceso comienza con la congelación del alimento, a una velocidad lenta, donde se promueve la formación de cristales grandes, facilitando la eliminación de la humedad, pero afecta la estructura de la membrana celular del vegetal; mientras una velocidad rápida, minimiza el daño en la estructura, por la formación de cristales pequeños, según lo reportado por Orrego (2008), de igual manera, cuando se congela por métodos criogénicos, pero se requiere más tiempo en el secado (Silva et al. 2016). Después, sigue el calentamiento, para retirar el agua por sublimación, en dos fases de secado; esto ocurre cuando la presión de vapor y la temperatura de la superficie del hielo están por debajo del punto triple, a $611,73 \mathrm{~Pa}$ y a $273,16^{\circ} \mathrm{K}$; aproximadamente, el $90 \%$ del agua se elimina en la primera fase (Serna et al. 2015). Las velocidades y los tiempos en el secado puede influir en las características físico-químicas y funcionales del producto final, según lo reportado por Silva et al. (2016) y estas condiciones de operatividad en la deshidratación de frutas tiene un impacto significativo en la estabilidad de los compuestos antioxidantes y fenólicos (M'hiri et al. 2017).

El objetivo de esta investigación fue evaluar el efecto de la liofilización sobre las propiedades funcionales del ají rocoto (Capsicum pubescens) con y sin semillas, variando la velocidad de calentamiento durante la sublimación, con el fin de evitar la pérdida de la capacidad antioxidante, fenoles totales, carotenoides, contenido de ácido ascórbico y capsaicina.

\section{MATERIALES Y MÉTODOS}

Material vegetal: Frutos de ají rocoto (Capsicum pubescens), colectados con igual índice de madurez de $11,12 \pm 2,98$, en la zona rural del corregimiento de San Cristóbal, Medellín - Antioquia (Colombia), con coordenadas geográficas de 75,63 Longitud (O) y 6,28 Altitud (N), a una altitud entre 1.800 y $2.200 \mathrm{msnm}$, con temperatura entre 8 y $21^{\circ} \mathrm{C}$, precipitación promedio anual de $1.668 \mathrm{~mm}$ y humedad relativa (HR) de $79 \%$. Los frutos fueron transportados hasta la Universidad Nacional de Colombia, sede Medellín y almacenados en el Laboratorio de Frutas y Hortalizas, a $5^{\circ} \mathrm{C}$ y $90 \%$ de HR; después, fueron lavados y desinfectados con hipoclorito de sodio a $80 \mathrm{ppm}$; se trabajó con un total de $30 \mathrm{~kg}$ de material vegetal.

Equipo de liofilización: Se utilizó un equipo Free Zone Stoppering Tray Dryer, Labconco, modelo 7948040 , de $12 \mathrm{~L}$ de capacidad volumétrica y tres bandejas, equipado con unidad de congelación y secado, con una bomba rotatoria de vacío de 195L/min y una presión de vacío de $14 \mathrm{~Pa}$; inicialmente, el material vegetal fue enfriado desde 25 a $-40^{\circ} \mathrm{C}$, con una velocidad de congelación de $0,10^{\circ} \mathrm{C} / \mathrm{min}$, para un tiempo total de $10,83 \mathrm{~h}$.

Preparación de los tratamientos: Para determinar el efecto de la liofilización sobre las propiedades funcionales del ají rocoto, se estudió el factor velocidad de calentamiento, durante la sublimación de las placas correspondiente a uno, tres y cinco segmentos de calentamiento, con una tasa de aumento de temperatura, entre 0,02 y $0,05^{\circ} \mathrm{C} / \mathrm{min}$ y un tiempo de sostenimiento entre cada uno de los segmentos, de 1,2h y con una temperatura final de $30^{\circ} \mathrm{C}$, por $3 \mathrm{~h}$. 
El otro factor de estudio fue el efecto de la semilla en los tratamientos con dos niveles: presencia de semillas (C) y ausencia de semillas (S). A los frutos, se les retiraron los pedúnculos y fueron troceado en tamaño de partícula de $10 \pm 2 \mathrm{~mm}$ de longitud, $10 \pm 2 \mathrm{~mm}$ de ancho y con $5 \pm 1 \mathrm{~mm}$ de espesor, incluyendo la epidermis; los tratamientos con semilla in- cluían la placenta del fruto, con un peso total de $425 \pm 0,5$ $\mathrm{g}$, por unidad experimental (UE); posteriormente, se dispusieron en bandejas de acero inoxidable y, a cada una, se les midió la temperatura, por termopar tipo K. En la tabla 1, se presenta el resumen de los tratamientos.

Tabla 1. Descripción de los tratamientos del ají rocoto (Capsicum pubescens) liofilizado.

\begin{tabular}{|c|c|c|c|c|}
\hline Parcela Principal & Subparcela & $\begin{array}{c}\text { Velocidad de } \\
\text { calentamiento } \\
\left({ }^{\circ} \mathbf{C} / \mathbf{m i n}\right)\end{array}$ & Tratamientos & Tiempo (h)* \\
\hline Programación 1 $\left(\mathrm{P}_{1}\right)$ & Con semilla $(\mathrm{C})$ & 0,05 & $\mathrm{P}_{1} \mathrm{C}$ & 26,3 \\
\hline Programación 1 $\left(\mathrm{P}_{1}\right)$ & Sin Semilla $(\mathrm{S})$ & 0,05 & $\mathrm{P}_{1} \mathrm{~S}$ & 26,3 \\
\hline Programación 2 $\left(\mathrm{P}_{2}\right)$ & Con semilla $(\mathrm{C})$ & 0,04 & $\mathrm{P}_{2} \mathrm{C}$ & 34,6 \\
\hline Programación 2 $\left(\mathrm{P}_{2}\right)$ & Sin Semilla $(\mathrm{S})$ & 0,04 & $\mathrm{P}_{2} \mathrm{~S}$ & 34,6 \\
\hline Programación 3 $\left(\mathrm{P}_{2}\right)$ & Con semilla $(\mathrm{C})$ & 0,02 & $\mathrm{P}_{3} \mathrm{C}$ & 45,3 \\
\hline Programación 3 $\left(\mathrm{P}_{2}\right)$ & Sin Semilla $(\mathrm{S})$ & 0,02 & $\mathrm{P}_{3} \mathrm{~S}$ & 45,3 \\
\hline
\end{tabular}

*Tiempo requerido para alcanzar un porcentaje de humedad 8,16 $\pm 1,62$ (b.s.).

Después que las muestras se liofilizaron fueron sometidas a molienda, en un equipo Fritsch Germany, con un tamaño de malla de $1 \mathrm{~mm}$, a $8.000 \mathrm{rpm}$, durante $5 \mathrm{~min}$, obteniendo un tamaño de partícula, aproximado, de $50 \mu \mathrm{m}$ y fueron almacenadas en bolsas de polietileno de baja densidad, selladas, rotuladas y almacenadas en frascos de vidrio ámbar, sellados herméticamente y guardados a $25^{\circ} \mathrm{C}$, para su posterior uso en las diferentes pruebas de laboratorio.

Preparación de las muestras para la determinación de la capacidad antioxidante y fenoles totales: Para los tratamientos liofilizados, se pesaron $0,3 \mathrm{~g}$ de ají rocoto y se adicionaron $10 \mathrm{~mL}$ de solvente $60 \%$ de etanol (Merck), durante $1 \mathrm{~min}$ se agitó, en un vortex mixer Fisher Scientific y se centrifugó en un equipo Hermle, modelo Z 206A, a 5.000rpm, durante $5 \mathrm{~min}, \mathrm{a} 23^{\circ} \mathrm{C}$. El sobrenadante fue recuperado y almacenado a $-25^{\circ} \mathrm{C}$, para su posterior análisis de capacidad antioxidante, por el método Ferric Reducing Antioxidant Power (FRAP) y contenido de fenoles totales.

Respecto al producto en fresco, se prepararon $2,9 \mathrm{~g}$, conservando la misma proporción en materia seca y participación de semilla que en los tratamientos liofilizados; fueron homogenizados, por 10s, en un procesador Ultrax-Turrax, de acuerdo con la metodología propuesta por(Wangcharoen \& Morasuk (2009).

Capacidad antioxidante por el método FRAP: Se realizó según la metodología modificada de (Zapata et al. (2014). El reactivo FRAP Sigma-Aldrich, St. Louis, MO, EE.UU. contie- ne 2,5mL de 2,4,6 - tripyridil -s- triazina (TPTZ) de concentración de $10 \mathrm{mM}$ en HcL 40Mm; 2,5mL de FecL3 20mM y $25 \mathrm{~mL}$ de tampón de acetato a pH 3,6 (0,3M). Se utilizaron $900 \mu \mathrm{L}$ de esta solución, $50 \mu \mathrm{l}$ de muestra y $50 \mu \mathrm{l}$ de agua destilada, a los 30 min se midió la absorbancia por triplicado, a una longitud de onda de 593nm, con respecto a un blanco. Las actividades de las muestras, se expresaron como AEAC Capacidad antioxidante en equivalentes de ácido ascórbico y se expresaron en mg de ácido ascórbico/g base seca.

Fenoles totales: La determinación, se realizó por el método colorimétrico modificado por López et al. (2016); 50 $\mu \mathrm{L}$ de muestra fueron mezclados con $125 \mu \mathrm{L}$ del reactivo Folinciocalteu Merck Millipore, Darmstadt y $400 \mu \mathrm{L}$ de carbonato de sodio de $7,1 \%$ p/v y, la solución restante, se llevó a un volumen final de $1000 \mu \mathrm{L}$. La mezcla se agitó y se almacenó a temperatura ambiente, durante 30min, en oscuridad. La absorbancia, se midió por triplicado a 760nm frente a un blanco, se usó la curva de referencia de las soluciones acuosas de ácido gálico, los resultados fueron expresados como mg de equivalente ácido gálico/g base seca.

Carotenoides: Se prepararon $15 \mathrm{mg}$ de ají rocoto liofilizado, se le adicionaron $5 \mathrm{~mL}$ de acetona (Merck), durante $2 \mathrm{~min}$ se agitó en un vortex mixer Fisher Scientific, se dejó reposar por $30 \mathrm{~min}$, a $4^{\circ} \mathrm{C}$, se centrifugó en un equipo HERMLE, modelo Z-206A, a $4.000 \mathrm{rpm}$, durante $10 \mathrm{~min}$, a $23^{\circ} \mathrm{C}$. Para el ají fresco, se tomaron $1,4 \mathrm{~g}$ de vegetal, conservando la misma proporción de materia seca y participación de semillas, que en los tratamientos liofilizados. El sobrenadante, se recuperó 
y se llevó a una celda fotométrica de vidrio Fisher Scientific, usando acetona como blanco. Se determinó la absorbancia de la solución a 449nm y se utilizó como estándar $\beta$-caroteno Sigma-Aldrich St. Louis, MO, EE.UU, de acuerdo a la metodología de Biswas et al. (2011).

Ácido ascórbico - vitamina C: Para la extracción del producto liofilizado, se pesó $1 \mathrm{~g}$ y se le adicionaron $10 \mathrm{~mL}$ de la solución de ácido metafosfórico; luego, se llevó a agitación en un vortex Boeco-Germany V1 plus, durante 1min y a un baño ultrasónico Bronson, modelo 3510, por 5min; se centrifugó en un equipo HETTICH, modelo Universal 320R, a $6.000 \mathrm{rpm}$, durante $10 \mathrm{~min}$, a $25^{\circ} \mathrm{C}$. Para el producto en fres$\mathrm{co}$, se pesaron $4 \mathrm{~g}$ de ají en una balanza analítica OHAUS, modelo PA2 14 y se le adicionó 30mL de ácido metafosfórico $\left(\mathrm{HPO}_{3}\right)$ Merck, Germany, con una pureza de 40 y $4 \% \mathrm{p} / \mathrm{v}$. La mezcla fue homogenizada y adicionada a tubos falcón de $50 \mathrm{~mL}$ y sometidos a baño de ultrasonido, por $5 \mathrm{~min}$; se centrifugó a $3.000 \mathrm{rpm}$, durante $10 \mathrm{~min}$, a $25^{\circ} \mathrm{C}$. Finalmente, el extracto obtenido, se filtró con membrana de nitrocelulosa Merck, Millipore Darmstadt, Alemania de 0,45 $\mu \mathrm{m}$ y se recolectaron $2 \mathrm{~mL}$ en un vial ámbar, para inyectar en el HPLC, según la metodología de Ruiz et al. (2010).

Las muestras, se analizaron en un cromatógrafo de líquidos de alta resolución HPLC - UFLC-Shimadzu, con un detector de diodos (PDA), columna en fase reserva C18(2), Luna $5 \mu \mathrm{m}$ C18(2) 100A Phenomenex, tamaño de partícula de 4,6mm x $250 \mathrm{~mm}$, la fase móvil fue de $0,2 \mathrm{M} \mathrm{KH}_{2} \mathrm{PO}_{4}$, ajustado su valor de pH final de 3,01 con ácido o-fosfórico al 85\%, con una velocidad de flujo $1,0 \mathrm{~mL} / \mathrm{min}$ y volumen de inyección de $5 \mu \mathrm{L}$. Para la determinación y la cuantificación, se construyó la curva de calibración, con un estándar de ácido ascórbico Supelco, Lote: LC0592V, USA, con el 99\% de pureza. La lectura, se realizó a $\lambda=244 \mathrm{~nm}$ y un tiempo de retención de $2,14 \pm 0,01 \mathrm{~min}$.

Capsaicina: Para la extracción del producto liofilizado, se pesaron $0,5 \mathrm{~g}$ en tubos de ensayo, recubiertos de papel aluminio, se le adicionaron $5 \mathrm{~mL}$ de acetonitrilo $\left(\mathrm{CH}_{3} \mathrm{CN}\right)$ J.T. Baker, pureza de 99,9\% grado HPLC y se agitaron en vortex Velp Scientific, modelo classic advanced, por 1min; seguidamente, se calentaron en baño maría Memmert, modelo WNB 14 a $60^{\circ} \mathrm{C}$ y con agitación constante, por 30min, hasta completar un total de $5 \mathrm{~h}$. Finalmente, se llevaron los tubos a refrigeración, para su posterior análisis. El sobrenadante obtenido, se filtró con membrana de nylon Merck Millipore de $0,45 \mu \mathrm{m}$ y se recogieron $2 \mathrm{~mL}$, en un vial ámbar, para inyectar en el cromatógrafo, de acuerdo con la metodología de(Cruz et al. (2007).

Para la extracción del producto en fresco, se pesaron $4 \mathrm{~g}$ de muestra, previamente picado, macerado y homogenizado, en un equipo GARRITY, Modelo G1138426, en tubos falcon de
$50 \mathrm{~mL}$, recubiertos en papel aluminio, para protegerlos de la acción de la luz. Se adicionaron $10 \mathrm{~mL}$ de acetona $\left(\mathrm{CH}_{3}\right) 2 \mathrm{CO}$ J.T. Baker, pureza 99,93\%; seguidamente, se agitó en vortex, por $1 \mathrm{~min}$. Se mantuvieron las muestras a temperatura de $25^{\circ} \mathrm{C}$, por tres días, para su posterior análisis. El sobrenadante fue separado con una jeringa y, finalmente, se filtró con membrana de nylon Merck Millipore de $0,45 \mu \mathrm{m}$ y se recuperaron $2 \mathrm{~mL}$ en un vial ámbar, para inyectar, inmediatamente al HPLC, de acuerdo con metodología de(Kollmannsberger et al. (2011).

Las muestras, se analizaron en un cromatógrafo de líquido de alta resolución (HPLC) UFLC-Shimadzu, con un detector de diodos - PDA, columna en fase reserva, luna $5 \mu \mathrm{m}$ C18(2) 100A Phenomenex, tamaño de partícula de 4,6mm x 250mm, la fase móvil fue Acetonitrilo:Agua 45:55, con una velocidad de flujo $1,0 \mathrm{~mL} / \mathrm{min}$ y volumen de inyección de $5 \mu \mathrm{L}$. La corrida, se realizó a $28 \pm 0,5^{\circ} \mathrm{C}$.

Para la cuantificación, se construyó una curva de calibración de un estándar de capsaicina Sigma-Aldrich, Lote: BCBP7674V, USA del 99,4\% de pureza y la lectura, se realizó a $\lambda=280 \mathrm{~nm}$ y un tiempo de retención de 21,52 $\pm 0,09 \mathrm{~min}$. Se elaboró una curva de calibración de puntos independientes, a partir de una solución madre de $1 \mathrm{mg} / \mathrm{mL}$, disuelto en acetonitrilo grado HPLC. Las soluciones fueron empacadas en frascos ámbar y almacenadas a temperatura de refrigeración $\left(4^{\circ} \mathrm{C}\right)$.

Diseño experimental y análisis de los datos: Se estructuró un arreglo de parcelas dividas, distribuidas completamente al azar (DCA), con tres repeticiones para el factor de la programación de la velocidad, asignado a la parcela principal $\left(\mathrm{P}_{1}\right.$, $\mathrm{P}_{2}$ y $\mathrm{P}_{3}$ ) y cuatro para el factor categórico en la subparcela, en los niveles con semilla (C) y sin semilla (S), para un total de 24 UE. Los resultados fueron expresados en promedio \pm la desviación estándar (DE). Se realizó un análisis de varianza (ANOVA), con el fin de determinar diferencias estadísticas entre los tratamientos realizados.

En el caso de encontrar diferencias entre los tratamientos, se procedió a realizar una prueba de comparación de medias de diferencia mínima significativa (DMS), con un nivel de significancia $\alpha<0,05$. Para las variables respuesta de los tratamientos liofilizados, se realizaron comparaciones de medias de dos poblaciones normales, con varianza heterogénea, con el producto en fresco con semilla (FC) y fresco sin semilla (FS), para $n=4$. Para todo el análisis estadístico, se empleó el programa estadístico R.

\section{RESULTADOS Y DISCUSIÓN}

Caracterización funcional del fruto fresco con semillas y sin semillas: En la tabla 2, se presentan las concentraciones 
obtenidas de las propiedades evaluadas en el ají rocoto ( $C$. pubescens) en fresco con semillas (FC) y sin semillas (FS). Se encontró en los tratamientos FC y FS, que la capacidad antioxidante, por el método FRAP y en fenoles totales, no se presentó una diferencia estadística $(\alpha>0,05)$, aunque se evidenció poca contribución. Gurnani et al. (2016) indican que las semillas del ají (C. frutescens L.) proporcionan fracciones contenidos de antioxidantes. Según lo reportado por Wangcharoen \& Morasuk (2009), para el ají (C. frutescens
L.), la capacidad antioxidante, por el método FRAP, fue de 7,11 mg, equivalentes de ácido ascórbico/g base seca, superior a la encontrada en la presente investigación, para ( $C$. pubescens). En fenoles totales, según Ornelas et al. (2010) y Vera et al. (2011) encontraron en el ají rocoto (C. pubescens) concentraciones entre 10,6 y 12,6mg, equivalentes de ácido gálico/g base seca, mientras que Loizzo et al. (2015) reportan valores de 5,81 mg/g base seca, para el ají (C. annuum), lo cual, es similar con lo encontrado.

Tabla 2. Capacidad antioxidante por FRAP, fenoles totales, carotenoides, vitamina C y capsaicina del ají rocoto (Capsicum pubescens), fresco con y sin semilla.

\begin{tabular}{|c|c|c|c|c|c|}
\hline Tratamiento & $\begin{array}{c}\text { Capacidad } \\
\text { antioxidante } \\
\text { (mg de ácido } \\
\text { ascórbico/g b.s.) }\end{array}$ & $\begin{array}{c}\text { Fenoles } \\
\text { (mg de ácido } \\
\text { gálico/g b.s.) }\end{array}$ & $\begin{array}{c}\text { Carotenoides } \\
\text { (mg de } \boldsymbol{\beta}- \\
\text { caroteno/g } \\
\text { b.s.) }\end{array}$ & $\begin{array}{c}\text { Ácido } \\
\text { ascórbico } \\
\text { (mg/g b.s.) }\end{array}$ & $\begin{array}{c}\text { Capsaicina } \\
\text { (mg/g b.s.) }\end{array}$ \\
\hline $\begin{array}{c}\text { Fresco con Semilla } \\
\text { (FC) }\end{array}$ & $1,80 \pm 0,03^{\mathrm{a}}$ & $5,37 \pm 0,03^{\mathrm{a}}$ & $4,74 \pm 0,02^{\mathrm{a}}$ & $1,18 \pm 0,16^{\mathrm{a}}$ & $1,57 \pm 0,09^{\mathrm{a}}$ \\
\hline $\begin{array}{c}\text { Fresco sin Semilla } \\
\text { (FS) }\end{array}$ & $1,12 \pm 0,06^{\mathrm{a}}$ & $5,13 \pm 0,08^{\mathrm{a}}$ & $2,79 \pm 0,08^{\mathrm{b}}$ & $1,44 \pm 0,13^{\mathrm{a}}$ & $0,20 \pm 0,01^{\mathrm{b}}$ \\
\hline
\end{tabular}

Los valores representan la media de $n=4 \pm$ la desviación estándar, (DMS, $\alpha<0,05$ ).

En el contenido de carotenoides, se evidenció diferencia significativa $(\alpha<0,05)$ entre los tratamientos FC y FS, con valores superiores en FC, muy posible a la presencia de estos compuestos en la semilla y en la placenta, de acuerdo a lo hallado por Simonovska et al. (2014), cuya contribución de contenido de carotenoides en la composición C. annuum L. en el pericarpio es de 0,42\% y en la placenta, de 0,32\%. En Oxaca, México, se reportaron valores de carotenoides, en la especie ají (C. pubescens), de $0,32 \mathrm{mg} / \mathrm{g}$ base seca (Vera et al. 2011), mientras que Rodríguez et al. (2010) reportaron concentraciones de 8,87 y 22,85mg/g base seca, en Bolivia.

En el contenido de ácido ascórbico no se evidenció diferencia significativa $(\alpha>0,05)$ entre FC y FS; algunos estudios reportan concentraciones de 15,2 a $38,1 \mathrm{mg} / \mathrm{g}$ base seca, en regiones de Puebla, México (Cruz et al. 2007); de 1,42mg/g b.s., en regiones de Perú (García et al. 2009) y de 1,71 a $2,19 \mathrm{mg} / \mathrm{g}$ b.s. en México (Vera et al. 2011), lo cual, indica que estas variaciones son posibles debido a la interacción del genotipo con el ambiente y, muy probablemente, a las prácticas culturales usadas en el cultivo y a las condiciones del suelo.

Para el contenido de capsaicina en el ají (C. pubescens), se obtuvo contenidos de $1,57 \pm 0,09 \mathrm{mg} / \mathrm{g}$ base seca en FC; se reportan en investigaciones concentraciones entre de 1,23 a 10,57 mg/g base seca (Meckelmann et al. 2015), otros reportan concentraciones de $1,47 \mathrm{mg} / \mathrm{g}$ base seca (Ornelas et al. 2010), resultados muy similares a los obtenidos en esta investigación.
Además, se evidenció una diferencia estadística $(\alpha<0,05)$ en las concentraciones de algunos compuestos evaluados para los tratamientos FC y FS, debido, posiblemente, a los compuestos fitoquímicos presentes en el arilo que recubre la semillas y en la misma semilla, como en el caso de la capsaicina, presente en el arilo y la secreción producida por la placenta, encontrando un alto contenido de este compuesto (Simonovska et al. 2014). Algunos estudios indican que el contenido de la capsaicina para $C$. pubescens oscila entre el 15 y 43\%, mientras que de dihidrocapsaicina, entre el 31 y $32 \%$, de los capsaicinoides totales (Kollmannsberger et al. 2011; Meckelmann et al. 2015) excepción a otras especies de Capsicum, donde se considera la capsaicina como el compuesto mayoritario (Ornelas et al. 2010).

Evaluación de las propiedades funcionales de los tratamientos liofilizados: En la tabla 3, se muestran los contenidos de la capacidad, los fenoles totales y los carotenoides evaluados en los tratamientos del ají rocoto liofilizado, en donde no se evidencia diferencia estadística $(\alpha>0,05)$ para la capacidad antioxidante; sin embargo, sí existe diferencia con respecto a los tratamientos FC y FS, presentándose un aumento significativo en la capacidad antioxidante y en la concentración de los fenoles totales en los tratamientos liofilizados, con respecto a los frutos frescos. Se obtuvo un contenido en fenoles totales en la programación dos con semillas $\left(\mathrm{P}_{2} \mathrm{C}\right)$, de 9,55 $\pm 0,16 \mathrm{mg}$, equivalente de ácido gálico/g base seca, tal como se presenta en la tabla 3 . De acuerdo con la clasificación para compuestos fenólicos, se encuentra en un nivel 
alto de estos metabolitos, que se establece cuando la concentración de mg equivalente de ácido gálico/g base seca es mayor a 5 (Zapata et al. 2014); resultados similares, se reportan en ají deshidratado, a temperaturas entre 30 y $70^{\circ} \mathrm{C}$ (Wangcharoen \& Morasuk, 2009; Ornelas et al. 2010; Arslan \& Özcan, 2011). Además, según lo indicado por Pérez et al. (2011), la liofilización tiene un efecto positivo al liberar compuestos fenólicos en la matriz estructural del tejido vegetal facilitando su extracción y acorde con lo reportado Silva et al. (2016), al incrementar el contenido de fenoles y flavonoides en los tratamientos liofilizados.

Con respecto al contenido de carotenoides, se evidencia una pérdida en los tratamientos liofilizados comparados con los frescos con semilla (FC) y frescos sin semillas (FS), lo cual, puede ser, debido a la degradación enzimática de estos compuestos, que están almacenados en las vacuolas plasmáticas, según lo reportado por(Shofian et al. (2011), además no se presentó una diferencia estadística entre los efectos de la programación para las diferentes velocidades y las categorías ( $\mathrm{C}$ y S) en los contenidos de carotenoides, que se consideran los precursores de la vitamina A.
En la figura 1A, se muestra el contenido de ácido ascórbico para los tratamientos liofilizados, se evidencia diferencia estadística $(\alpha<0,05)$, en el efecto principal consistente en las programaciones de velocidad de calentamiento durante la sublimación, indicando para el tratamiento $\mathrm{P}_{2}$ las mayores concentraciones, aunque para el efecto categórico C y S, no se presentó diferencia estadística, con respecto a la proporción de los contenidos iniciales en frutos frescos con semillas (FC) y sin semillas (FS).

$\mathrm{Al}$ analizar el contenido de vitamina $\mathrm{C}$ entre los tratamientos liofilizados y los frescos no se evidenció diferencia estadística $(\alpha>0,05)$ entre las medias, indicando un efecto mínimo de deterioro, debido al proceso de liofilización, resultado que se encuentra acorde a lo reportado por Shofian et al. (2011). En el C. annuum L. detectó que contenía 1,8 veces más ácido ascórbico en los tratamientos liofilizados con respecto al secado por convección forzada con aire caliente a $60^{\circ} \mathrm{C}$ (Toontom et al. 2012). Otros estudios han evidenciado que los procesos de deshidratación por convección forzada, a temperaturas de secado de 45,55 y $65^{\circ} \mathrm{C}$, presentan pérdidas de vitamina C, entre un 32 y 68\%, en Capsicum sp. (Reis et al. 2013).

Tabla 3. Capacidad antioxidante por FRAP, fenoles totales y carotenoides del ají rocoto (Capsicum pubescens), de los tratamientos liofilizados.

\begin{tabular}{|c|c|c|c|c|}
\hline \multicolumn{2}{|c|}{ Propiedades } & $\mathbf{P}_{1}$ & $\mathrm{P}_{2}$ & $\mathrm{P}_{3}$ \\
\hline $\begin{array}{c}\text { Capacidad antioxidante } \\
\text { (mg de ácido ascórbico/g b.s) }\end{array}$ & $\mathrm{C}$ & $2,34 \pm 0,47^{\mathrm{a}}$ & $3,38 \pm 0,58^{\mathrm{a}}$ & $2,99 \pm 0,61^{\mathrm{a}}$ \\
\cline { 2 - 5 } & $\mathrm{S}$ & $3,41 \pm 0,60^{\mathrm{a}}$ & $3,03 \pm 0,86^{\mathrm{a}}$ & $2,68 \pm 1,03^{\mathrm{a}}$ \\
\hline \multirow{2}{*}{$\begin{array}{c}\text { Fenoles } \\
\text { (mg de ácido gálico / g b.s) }\end{array}$} & $\mathrm{C}$ & $7,21 \pm 0,94^{\mathrm{b}}$ & $9,55 \pm 0,16^{\mathrm{a}}$ & $7,04 \pm 0,97^{\mathrm{b}}$ \\
\cline { 2 - 5 } & $\mathrm{S}$ & $8,66 \pm 0,76^{\mathrm{a}}$ & $9,03 \pm 0,60^{\mathrm{a}}$ & $8,84 \pm 0,92^{\mathrm{a}}$ \\
\hline $\begin{array}{c}\text { Carotenoides } \\
\text { (mg de } \beta \text { - caroteno / g b.s) }\end{array}$ & $\mathrm{C}$ & $1,22 \pm 0,19^{\mathrm{a}}$ & $1,05 \pm 0,57^{\mathrm{a}}$ & $0,83 \pm 0,44^{\mathrm{a}}$ \\
\cline { 2 - 5 } & $\mathrm{S}$ & $1,31 \pm 0,47^{\mathrm{a}}$ & $0,87 \pm 0,50^{\mathrm{a}}$ & $1,48 \pm 0,60^{\mathrm{a}}$ \\
\hline
\end{tabular}

Los valores representan la media de $n=4 \pm$ la desviación estándar, (DMS, $\alpha<0,05)$.

En la figura 1B, se presenta el contenido de capsaicina de los tratamientos liofilizados, donde se hallaron diferencias estadísticas, tanto debidas al efecto de la programación de la velocidad de calentamiento en la sublimación como en la categoría presencia o ausencia de semillas, viéndose una mayor concentración de capsaicina por la presencia de semillas, tanto para el producto en fresco como para los tratamientos liofilizados y, ello, probablemente, a la inclusión de la placenta, donde se encuentra gran cantidad de este metabolito, de acuerdo a lo reportado por Simonovska et al. (2014) . El efecto del tiempo y la velocidad durante el secado disminuyó las concentraciones finales de capsaicina en el producto, lo cual, está acorde con lo hallado por Yaldiz et al. (2010).
El contenido de capsaicina entre los tratamientos liofilizados y los frescos presentan diferencia estadística $(\alpha<0,05)$, debido a la pérdida de este componente en los tratamientos liofilizados; estas disminuciones en las concentraciones del metabolito, pueden ser, debido a la actividad catalítica de las enzimas como la peroxidasa que degrada el compuesto, lo cual, ha sido reportado por algunos investigadores, como(Topuz et al. (2011).

Se pudo concluir en esta investigación, que las condiciones de operación de la programación 2 en la liofilización, se obtuvo una mayor concentración de capsaicina en los vegetales liofilizados integralmente con semillas y placenta y, además, 

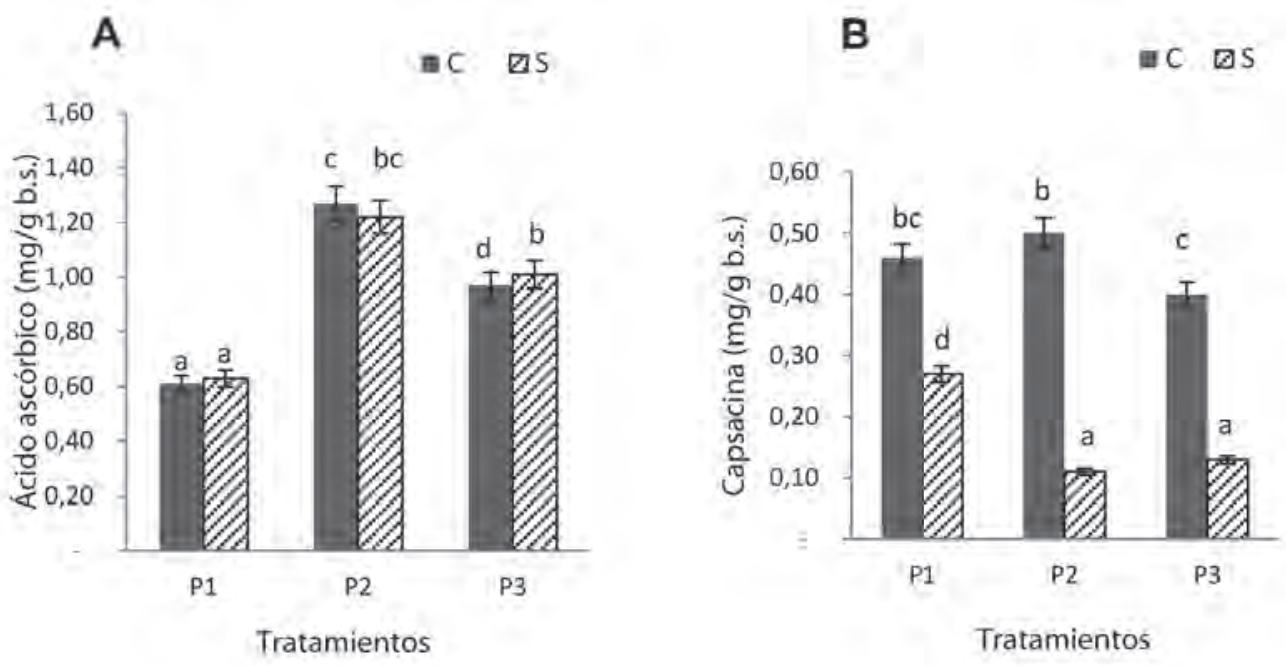

Figura 1. Contenido de ácido ascórbico: (A) y contenido de capsaicina (B) de los tratamientos de ají rocoto (Capsicum pubescens) liofilizado con semillas $(C)$ y sin semillas $(S)$, para cada una de las programaciones $\left(P_{1}, P_{2}, P_{3}\right)(D M S, \alpha<0,05)$ para $n=4$.

permitió conservar la concentración de ácido ascórbico con respecto a los productos frescos. El ají rocoto liofilizado contiene niveles importantes de compuestos funcionales, como fenoles totales, ácido ascórbico y capsaicina.

Agradecimientos: Los autores agradecen al personal técnico de los laboratorios de futas y hortalizas, control de calidad de alimentos y ciencias de los alimentos de la Universidad Nacional de Colombia, sede Medellín. Conflicto de intereses: El manuscrito fue preparado y revisado con la participación de todos los autores, quienes declaramos que no existe conflicto de interés que pongan en riesgo la validez de los resultados presentados.

\section{BIBLIOGRAFÍA}

1. ARSLAN, D.; ÖZCAN, M. 2011. Dehydration of red bellpepper (Capsicum annuum L.): Change in drying behavior, colour and antioxidant content. Food and Bioproducts Processing. (United Kingdom). 89(4):504-513.

2. BISWAS, A.K.; SAHOO, J.; CHATLI, M.K. 2011. A simple UV-Vis spectrophotometric method for determination of $\beta$-carotene content in raw carrot, sweet potato and supplemented chicken meat nuggets. LWT - Food Sc. and Tech. (United States). (44(8):18091813.

3. BORT, A.; MORELL, M.C.; RAMOS, Á.; DELGADO, L.; DIAZ, I.; RODRÍGUEZ, N. 2014. Efecto de la capsai- cina en el metabolismo de células de hepatocarcioma. Dianas. (España). 3(1):1-9.

4. CRUZ, A.; GONZÁLEZ, V.; SOTO, R.; GUTIÉRREZ, M.; GARDEA, A.; PERÉZ, M. 2007. Capsaicinoides, vitamina $\mathrm{C}$ y heterosis durante el desarrollo del fruto de chile manzano. Agrociencia. (México). 41:627-635.

5. GARCÍA, M.; GÓMEZ, I.; ESPINOZA, C.; BRAVO, F.; GANOZA, L. 2009. Tablas peruanas de composición de alimentos. Ministerio de Salud, Centro Nacional de Alimentación y Nutrición (Lima). p.22-23.

6. GURNANI, N.; GUPTA, M.; MEHTA, D.; MEHTA, B. 2016. Chemical composition, total phenolic and flavonoid contents, and in vitro antimicrobial and antioxidant activities of crude extracts from red chilli seeds (Capsicum frutescens L.). J. Taibah University for Science (Saudi Arabia). 10(4):462-470.

7. HALIKOWSKI, S. 2015. In the shadow of a pepper-centric historiography: Understanding the global diffusion of capsicums in the sixteenth and seventeenth centuries. J. Ethnopharmacology (Ireland). 167:6477.

8. KOLLMANNSBERGER, H.; RODRÍGUEZ, A.; NITZ, S.; NUEZ, F. 2011. Volatile and capsaicinoid composition of ají (Capsicum baccatum) and rocoto (Capsicum pubescens), two andean species of chile peppers. J Sc Food Agri. (United Kingdom). 91(9):1598-1611. 
9. LOIZZO, M.R.; PUGLIESE, A.; BONESI, M.; MENICHINI, F.; TUNDIS, R. 2015. Evaluation of chemical profile and antioxidant activity of twenty cultivars from Capsicum annuum, Capsicum baccatum, Capsicum chacoense and Capsicum chinense: A comparison between fresh and processed peppers. LWT - Food Sc and Tech. (United States). 64(2):623-631.

10. LÓPEZ, E.; PILATOWSKY, I.; CORTÉS, F.; ROJANO, B.; NAVARRO, A. 2016. Effect of temperature on antioxidant capacity during drying process of mortiño (Vaccinium meridionale Swartz). Int. J. Food Properties. (United States). 2912(3):1532-2386.

11. MECKELMANN, S.W.; JANSEN, C.; RIEGEL, D.W.; ZONNEVELD, M.; RÍOS, L.; PEÑA, K.; PETZ, M. 2015. Phytochemicals in native peruvian Capsicum pubescens (Rocoto). European Food Res and Tech. (Germany). 241(6):817-825.

12. M'HIRI, N.; IOANNOU, I.; GHOUL, M.; MIHOUBI, N. 2017. Phytochemical characteristics of citrus peel and effect of conventional and nonconventional processing on phenolic compounds: A review. Food Reviews International. (United States). 33(6):587-619.

13. ORNELAS, J.; MARTINÉZ, J.M.; RUIZ, S.; SANTANA, V.; IBARRA, V.; OLIVAS, G.; PÉREZ, J.D. 2010. Effect of cooking on the capsaicinoids and phenolics contents of mexican peppers. Food Chemistry. (Netherlands) 119(4):1619-1625.

14. ORREGO, C. 2008. Congelación y Liofilización de Alimentos. Universidad Nacional de Colombia. (Colombia).172p.

15. PÉREZ, M.; REGUEIRO, J.; GONZÁLEZ, C.; RIAL, R.; SIMAL, J. 2011. Changes in antioxidant flavonoids during freeze-drying of red onions and subsequent storage. Food Control. (Netherlands). 22(7):11081113.

16. REIS, R.C.; CASTRO, V.C.; DEVILLA, I.A.; OLIVEIRA, C.A.; BARBOSA, L.S.; RODOVALHO, R. 2013. Effect of drying temperature on the nutritional and antioxidant qualities of cumari peppers from pará; (Capsicum chinense Jacqui). Brazilian J. Chemical Eng. 30(2):337-343.

17. RODRÍGUEZ, A.; GONZÁLEZ, C.; NUEZ, F. 2010. Carotenoid composition and vitamin A value in ají (Capsicum baccatum L.) and rocoto (C. pubescens R. \& P.), 2 pepper species from the Andean region. J. Food Sc. (United States). 75(8):S446-453.
18. RUIZ, S.; ÁLVAREZ, E.; ROSA, L.A; MARTÍNEZ, A.I.; ORNELAS, J.; MENDOZA, A.M.; GONZÁLEZ, G. 2010. Effect of Different Sanitizers on microbial, sensory and nutritional quality of fresh-cut jalapeno peppers. American J. Agricultural and Biological Sc. (United States). 5(3):331-341.

19. SERNA, L.; VARGAS, D.; AYALA, A. 2015. Structural, physical, functional and nutraceutical changes of freeze-dried fruit. African J. Biotechnology. (Nigeria). 14(6):442-450.

20. SHARMA, S.K.; VIJ, A.S.; SHARMA, M. 2013. Mechanisms and clinical uses of capsaicin. European $\mathrm{J}$. Pharmacology. (Netherlands). 720(1-3):55-62.

21. SHOFIAN, N.M.; HAMID, A.A.; OSMAN, A.; SAARI, N.; ANWAR, F.; DEK, M.S.P.; HAIRUDDIN, M.R. 2011. Effect of freeze-drying on the antioxidant compounds and antioxidant activity of selected tropical fruits. International J. Molecular Sc. (Switzerland). 12(7):4678-4692.

22 SILVA, N.; SANTANA, R.; DUARTE, C.; BARROZO, M. 2016. Impact of freeze-drying on bioactive compounds of yellow passion fruit residues. J. Food Process Engineering. (United States). 2016:1-9.

23. SIMONOVSKA, J.; RAFAJLOVSKA, V.; KAVRAKOVSKI, Z.; SRBINOSKA, M. 2014. Nutritional and bioactive compounds in hot fruits. Macedonian Journal of Chemistry and Chemical Engineering. (Macedonia). 33(1):97-104.

24. TOONTOM, N.; MEENUNE, M.; POSRI, W.; LERTSIRI, S. 2012. Effect of drying method on physical and chemical quality, hotness and volatile flavour characteristics of dried chilli. Intern Food Res. J. (Malaysia). 19(3):1023-1031.

25. TOPUZ, A.; DINCER, C.; ÖZDEMIR, K.S.; FENG, H.; KUSHAD, M. 2011. Influence of different drying methods on carotenoids and capsaicinoids of paprika (Cv. Jalapeño). Food Chemistry. (Netherlands). 129(3):860-865.

26. VERA, A.; CHÁVEZ, J.; CARRILLO, J.; LÓPEZ, M. 2011. Phytochemical evaluation of wild and cultivated pepper (Capsicum annuum L. and C. pubescens Ruiz \& Pav.) from Oaxaca, Mexico. Chilean J Agricultural Res. (Chile). 71:578-585.

27. WANGCHAROEN, W.; MORASUK, W. 2009. Antioxidant capacity changes of bird chili (Capsicum frutescens 
Linn.) during hot air drying. Kasetsart J. (Natural Science). (Thailand). 20:12-20.

28. YALDIZ, G.; OZGUVEN, M.; SEKEROGLU, N. 2010. Variation in capsaicin contents of different Capsicum species and lines by varying drying parameters. Industrial Crops and Products. (Netherlands). 32(3):434-438.
29. ZAPATA, S.; PIEDRAHITA, A.M.; ROJANO, B. 2014. Capacidad atrapadora de radicales oxígeno (ORAC) y fenoles totales de frutas y hortalizas de Colombia. Perspectivas en Nutrición Humana. (Colombia). 16(1):25-36.

Recibido: Febrero 23 de 2017

Aceptado: Mayo 30 de 2017

\section{Cómo Citar:}

Caballero Gutiérrez, B.L.; Márquez Cardozo, C.J.; Rojano, B.A. 2017. Efecto de la liofilización sobre las propiedades funcionales del ají rocoto (Capsicum pubescens). Rev. U.D.C.A. Act. \& Div. Cient. 20(1): 111-119. 